\title{
A LITERATURE REVIEW ON HYPERSPECTRAL IMAGE DENOISING
}

\author{
V Chandrasekhar, Shaik Taj Mahaboob \\ M.Tech Student, Assistant Professor \\ Electronics and Communication Engineering, \\ JNTUA College of Engineering Pulivendula, Pulivendula, India.
}

\begin{abstract}
Hyperspectral images are threedimensional images. These consist of two types of domains. They were spatial domain and spectral domain. Hyperspectral image contains very much contamination while capturing from a spectral camera. These images can be captured at a particular wavelength using the electromagnetic spectrum. To eliminate this noise various techniques are there. A few of them are 1. Global low-rank representation 2. Local low-rank representation, 3. Sparse representation etc. while eliminating this noise using various techniques, we can apply for many problems like target detection, material identification on the earth's surface, and agriculture field. Here we have discussed and analyzed with opposing approaches and existing strategies to solve the problems. Denoising approaches have thus been the key step toward developing object identification and classification in remote sensing imaging applications and military applications.
\end{abstract}

Index terms: Hyperspectral images, Global low-rank representation [8], Local low-rank representation [10], Sparse representation [10].

\section{INTRODUCTION}

Now a day, hyperspectral images play a significant position in the agriculture sector, medical, remote sensing, and military applications [1]. This picture consists an abundance of a block by block spectral and spatial scene detail. In real-life scenes due to minimal reasons like that dark current, changes to a power source, whereas capturing phase. Generally, hyperspectral images are contaminated by various noise and don't have clarity in the images to identify materials.

The various methods of denoising will give the result of the hyperspectral image into a matrix in which the columns contain spectral information of pixels. Because of the matrix-based techniques could not give the spectral information [4]. But present days denoising methods have been playing a critical role in the different fields. The denoising methods are nonlocal due to the principles, the averages can be calculated based on overall pixels of the images and some pixels can be grouped [6]. The paradigms in the denoising are classical and nonlocal based approaching methods.

There are number of Hyperspectral image-denoising methods used in the image processing. Sparse depiction and low-rank portrayal are used in the hyperspectral images to eliminate noise. Many of the above strategies make to use the global use of the low-ranked property worldwide. But here we must use this approach locally. However, adjacent pixels have strong correlations in both spatial and spectral ways, certain blocks of pixels, which are free from noise, that are belongs to HIS [3].

However, there are various noises applications of the hyperspectral images, we are using based on our needs like material identification, military, medicine and agriculture fields. So, denoising plays important role in this world. The techniques are manually for hyperspectral images.

\section{HYPERSPECTRAL IMAGE DENOSING TECHNIQUES}

i. Global low-rank representation: Global low-rank representation is a denoising technique which is mainly used for the hyperspectral images. Here, in this technique, we use an entire image for the denoising by applying the algorithms for the entire image [8]. This is the first technique that can be applied for the removal of noise present in the hyperspectral image denoising.

ii. Local low-rank representation: Local lowrank representation is an advanced technique to global low-rank representation [10]. Here, in this technique we consider an entire image to get our required image denoising image. This can be used for the removal of noise by taking several iteration methods. That is, it can be taken to the state-of-theart method. 


\section{International Journal of Engineering Applied Sciences and Technology, 2020 \\ Vol. 5, Issue 7, ISSN No. 2455-2143, Pages 248-250 \\ Published Online November 2020 in IJEAST (http://www.ijeast.com)}

iii. Sparse representation: Sparse representation is a technique that is using to DE-noise the hyperspectral images. It is used to convert high dimensional spectral into low dimensional [6]. Therefore, the image formed is free from abundant contamination in the spectral and spatial images. This technique is rottenly used to minimize the hyperspectral data that can be either in the form of spectral or spatial nature because of the features of the hyperspectral images [10].

iv. Convolutional neural network: convolutional neural network is a technique, which is used for, object or image recognition. This can be applicable where the pixels are the main objects to apply this network. It can be worked with the several layers that all layers are using to maintain visual clarity of the hyperspectral images, which are having enormously powerful vision to the object recognition. Due to hyperspectral images, having a greater number of bands it takes more time to get good resolution and clarity of the identifying object.

v. Principal component analysis: Principal component analysis is a technique where we use one step at a time using singular value decomposition (SVD). Shifting the data did not change how the data points are positioned relative to each other. It can be used for the selected blocks of pixels are used to remove the noise present in the images.

Table 1: A summary of hyperspectral image denoising techniques

\begin{tabular}{|c|c|c|c|c|}
\hline S. No & Title & Reference & $\begin{array}{l}\text { Method/algorithm } \\
\text { implemented }\end{array}$ & Advantage \\
\hline 1. & $\begin{array}{l}\text { The assessment of } \\
\text { independent } \\
\text { component analysis for } \\
\text { the detection of } \\
\text { military targets from } \\
\text { hyperspectral images. }\end{array}$ & D. Singh & $\begin{array}{l}\text { Independent } \\
\text { component analysis } \\
\text { (ICA) }\end{array}$ & $\begin{array}{c}\text { To denoise a linear } \\
\text { prediction of Gaussian } \\
\text { noise in the hyperspectral } \\
\text { photos. }\end{array}$ \\
\hline 2. & $\begin{array}{l}\text { The sparse } \\
\text { representation-based } \\
\text { band selection for } \\
\text { hyperspectral images }\end{array}$ & Hairong Qi & K-SVD algorithm & $\begin{array}{l}\text { To remove the sparseness } \\
\text { and noise in the } \\
\text { hyperspectral data. }\end{array}$ \\
\hline 3. & $\begin{array}{l}\text { The remote Sensing } \\
\text { Scene Classification by } \\
\text { Unsupervised } \\
\text { Representation } \\
\text { Learning }\end{array}$ & Xiangtan & Bottom-up method & $\begin{array}{c}\text { Reduces the difference } \\
\text { between functionality at } \\
\text { low levels and high-level } \\
\text { seamanship }\end{array}$ \\
\hline 4. & $\begin{array}{l}\text { Nonlocal transform- } \\
\text { domain Filter for } \\
\text { Volumetric } \\
\text { Data Denoising and } \\
\text { Reconstruction }\end{array}$ & Matteo & $\begin{array}{l}\text { Block matching } \\
\text { 3-Dimensional } \\
\text { (BM3D) }\end{array}$ & $\begin{array}{l}\text { Representatively, } \\
\text { reflecting the present state } \\
\text { of the art in 2-D image } \\
\text { denoising. }\end{array}$ \\
\hline 5. & $\begin{array}{l}\text { Local Low-Rank and } \\
\text { Sparse Representation } \\
\text { for Hyperspectral } \\
\text { Image Denoising. }\end{array}$ & Jie Huang & $\begin{array}{l}\text { Block matching } \\
\text { 4-Dimensional } \\
\text { (BM4D) }\end{array}$ & $\begin{array}{l}\text { To remove strip noise in } \\
\text { the hyperspectral data. }\end{array}$ \\
\hline
\end{tabular}

\section{CONCLUSION}

This paper provides a study of the framework of hyperspectral image denoising approaches that have been discussed and presents a literature survey on the various techniques involved in hyperspectral image denoising. These methods are measured based on denoising the hyperspectral image denoising based on various techniques using various algorithms and requested references are listed below. In denoising 


\section{International Journal of Engineering Applied Sciences and Technology, 2020 \\ Vol. 5, Issue 7, ISSN No. 2455-2143, Pages 248-250 \\ Published Online November 2020 in IJEAST (http://www.ijeast.com)}

methods, we can adopt a Convolutional Neural Network [CNN] [3] in the field of hyperspectral image analysis, to investigate non-local similarities and global low-rank properties.

- The number of iterations in the algorithm will reduce the noise that is present in the hyperspectral image as band-by-band. So, the performance of operation will become easier to eliminate various noise in the pixels.

- In addition, a modern low-rank approximation philtrum is invented for philtra noisy 3D-patches. The entrants outperformed consistently in a comparison with the state-of-the-art GLF. So, this is our key field to denoise in the analysis of hyperspectral images.

\section{ACKNOWLEDGEMENT}

I really thank the authors who have given us a basic knowledge on the study of hyperspectral image denoising methods. The local low-rank and sparse representation will become the two advance techniques which are used to denoise hyperspectral images.

\section{REFERENCES}

1. Tiwari K.C, Arora M.K, and Singh D, Oct 2011, "An assessment of independent component analysis for detection of military targets from hyperspectral images" Int. J. Appl. Earth Observ. Geoinf., vol. 13, no. 5, pp. 730740 .

2. Lu X, Zheng X, and Yuan Y, Sep.2011, "Remote sensing scene classification by unsupervised representation learning" IEEE Trans. Geosci. Remote Sens, vol. 55, no. 9, pp. 5148-5157. Maggioni M, Katkovnik V, Egiazarian K, and Foi A, Apr.2013 "Nonlocal transform-domain filter for volumetric data denoising and reconstruction" IEEE Trans. Image Process., vol. 22, no. 1, pp. 119-133.

3. Chen Y, Huang T.Z, Zhao X.L, and Deng L.J, Nov.2018 "Hyperspectral image restoration using framelet-regularized lowrank non-negative matrix factorization" Appl. Math. Model, vol. 63, pp. 128-147.
4. Do NoHo D.L, May 1995 "De-noising by soft-thresholding” IEEE Trans. Inf. Theory, vol. 41, no. 3, pp. 613-627.

5. $\mathrm{Hu}$ J, Li Y and Xie W, Oct 2017 "Hyperspectral image super-resolution by spectral difference learning and spatial error correction" IEEE Geosci. Remote Sens. Lett. vol. 14, no. 10, pp. 1825-1829.

6. Cao W, Wang Y, Sun J, Meng D, Yang C, Cichocki A, and Xu Z, Sep.2016 "Total variation regularized tensor RSPCA for background subtraction from compressive measurements" IEEE Trans. Image Process., vol. 25, no. 9, pp. 4075-4090.

7. Chen G and Qian S.E, Mar.2011 "Denoising of hyperspectral imagery using principal component analysis and wavelet shrinkage" IEEE Trans. Geosci. Remote Sens., vol. 49, no. 3, pp. 973-980.

8. He W, Zhang H, Zhang L, and Shen H, Jan.2016 "Total-variation-regularized lowrank matrix factorization for hyperspectral image restoration" IEEE Trans. Geosci. Remote Sens., vol. 54, no.1 pp. 178-188.

9. Zhao Y.Q and Yang J, Jan.2015 "Hyperspectral image denoising via sparse representation and low-rank constraint" IEEE Trans. Geosci. Remote Sens., vol. 53, no. 1, pp. 296308, Jan. 2015.

10. Qian Y and Ye M, Apr.2013 "Hyperspectral imagery restoration using nonlocal spectralspatial structured sparse representation with noise estimation," IEEE J. Sel. Topics Appl. Earth Observ. Remote Sens., vol. 6, no. 2, pp. 499-515.

11. Zhang H, He H.W, Zhang L, Shen L, and Yuan Q, Aug.2014 "Hyperspectral image restoration using low-rank matrix recovery," IEEE Trans. Geosci. Remote Sens., vol. 52, no. 8, pp. 4729-4743.

12. Zhou X and Yu W, Sep.2013 "Low-rank modeling and its applications in medical image analysis," in Proc. SPIE Defense Secur, p. 87500V.

13. Rasti B, Sveinsson J, Ulfarsson M, and Benediktsson J, "Hyperspectral image denoising using first order spectral roughness penalty in wavelet domain," IEEE J. Sel. Topics Appl. Earth Observ. Remote Sens., vol. 7, no. 6, pp. 2458-2467, Jun. 2014. 